\title{
Newer fluoroquinolones for treating respiratory infection: do they mask
} tuberculosis?

\author{
K.C. Chang*, C.C. Leung*, W.W. Yew*, T.Y. Lau*, W.M. Leung*, C.M. Tam*, \\ H.C. Lam*, P.S. Tse*, M.Y. Wong*, S.N. Lee*, K.I. Wat* and Y.H. Ma*
}

ABSTRACT: Possible masking of tuberculosis (TB) in treatment of community-acquired respiratory infection by newer fluoroquinolones has not been examined in randomised controlled trials.

We undertook a randomised, open-label controlled trial involving adults with communityacquired pneumonia or infective exacerbation of bronchiectasis encountered in government chest clinics in Hong Kong. 427 participants were assigned by random permutated blocks of 20 to receive either amoxicillin clavulanate $(n=212)$ or moxifloxacin $(n=215)$. Participants were followed for $1 \mathrm{yr}$ for active pulmonary TB.

Excluding three participants with positive baseline culture, 13 developed active pulmonary TB: $10(4.8 \%)$ out of 210 were given amoxicillin clavulanate, and three (1.4\%) out of 214 were given moxifloxacin. The difference was significant by both proportion and time-to-event analysis. Post hoc analysis showed a significant decrease in the proportion with active pulmonary TB from $4.8 \%$ to $2.4 \%$ and $0 \%$ among participants given amoxicillin clavulanate $(n=210)$, moxifloxacin for predominantly 5 days $(n=127)$ and 10 days $(n=87)$, respectively. The log rank test for trend also showed a significant difference between the three subgroups. Regression models reaffirmed the linear effect; the adjusted odds ratio ( $95 \%$ confidence interval) of active pulmonary TB after moxifloxacin exposure up to predominantly 10 days was $0.3(0.1-0.9)$.

Newer fluoroquinolones appear to mask active pulmonary TB.

\section{KEYWORDS: Fluoroquinolones, tuberculosis}

W ith broad-spectrum antimicrobial coverage including $\beta$-lactamase-producing bacteria, penicillin-resistant Streptococcus pneumoniae, and pathogens causing atypical pneumonia, newer fluoroquinolones have demonstrated effectiveness in treatment of community-acquired respiratory infection [1-6]. The Infectious Diseases Society of America and the American Thoracic Society have recommended newer fluoroquinolones for treating community-acquired pneumonia (CAP) in the presence of comorbidities or risk factors for drug-resistant $S$. pneumoniae infection [7]. Like older fluoroquinolones, which have demonstrated an important role in treatment of multidrug-resistant tuberculosis (TB) [8-11], newer fluoroquinolones have shown prominent anti-TB activity in the initial phase of TB treatment with potential for shortening treatment duration $[12,13]$

Possible masking of active TB in the treatment of $\mathrm{CAP}$ by fluoroquinolones has been suggested by retrospective analysis [14], but not been verified by randomised controlled trials in TB-endemic areas. Hong Kong is endemic for TB with annual notification rates at $\sim 80$ per 100,000 persons [15]. Patients with community-acquired respiratory infections including pneumonia, infective exacerbation of bronchiectasis and active TB are commonly managed in government chest clinics. Thus, we designed a clinical trial to test the null hypothesis that newer fluoroquinolones in the treatment of community-acquired respiratory infection would not mask active pulmonary TB.

\section{METHODS}

\section{Patients}

We undertook a randomised, open-label controlled trial involving patients with CAP or infective exacerbation of bronchiectasis encountered in multiple government chest clinics in Hong Kong. Adult patients aged $\geqslant 18$ yrs were

\section{AFFILIATIONS}

*Tuberculosis and Chest Service, Centre for Health Protection, Dept of Health, and

\#Tuberculosis and Chest Unit, Grantham Hospital, Hong Kong SAR, China.

\section{CORRESPONDENCE}

K.C. Chang

Wanchai Chest Clinic

1st Floor, Wanchai Polyclinic

99 Kennedy Road

Wanchai

Hong Kong

E-mail: kc_chang@dh.gov.hk

Received:

July 032009

Accepted after revision:

Aug 202009

First published online:

Aug 282009 
eligible for enrolment if they had clinical signs and symptoms of CAP or infective exacerbation of bronchiectasis with compatible findings on the chest radiograph. Symptoms and signs included one or more of the following: cough, sputum, haemoptysis, chest discomfort, shortness of breath, crackles and fever. Patients without symptoms were also eligible in the presence of opacities compatible with consolidations on the chest radiograph. Exclusion criteria were: age $<18$ yrs; confirmed or suspected pregnancy; positive baseline sputum acid-fast bacilli (AFB) smear or AFB culture, and when either amoxicillin clavulanate or moxifloxacin was inappropriate for the clinical scenario, such as a history of adverse events to amoxicillin clavulanate or moxifloxacin; significant renal impairment; or known congenital or acquired conditions associated with prolonged QTc interval, symptomatic cardiac failure or arrhythmia.

Participants underwent baseline clinical assessment including evaluation by chest radiography, sputum microscopy and bacteriology including AFB culture. Chest radiographs and sputum AFB bacteriology were taken at 6 and 12 months after enrolment and whenever they were considered necessary by the attending physician according to the clinical progress. Further investigations, which included complete blood picture, liver and renal biochemistry, computerised tomography of thorax, and fibreoptic bronchoscopy, could be arranged by the attending physician according to the clinical condition and progress to aid diagnosis or evaluate severity.

Written informed consent was obtained from study participants before enrolment. Approval for conducting the study was obtained from the Ethics Committee of the Dept of Health of Hong Kong Special Administrative Region.

\section{Procedures}

A study coordinator allocated eligible patients by random permutated blocks of 20 in equal proportions to one of two arms: moxifloxacin $400 \mathrm{mg}$ once daily for 5 days versus amoxicillin clavulanate $375 \mathrm{mg}$ twice daily plus amoxicillin $750 \mathrm{mg}$ twice daily for 5 days. Baseline dosing schedules were based on published literature about the efficacy of short-course antibiotic treatment of CAP [16]. The attending physician was advised not to find out the identity of trial antibiotics unless it was indicated by the clinical condition, such as severe adverse events. The attending physician could extend the duration of study antibiotics, repeat study antibiotics, or prescribe other antibiotics including fluoroquinolones subsequently, according to the clinical progress.

Participants were followed for 1 yr for active pulmonary TB, the primary end-point. Active pulmonary TB was diagnosed when compatible clinical findings coexisted with one or more of the following conditions: isolation of Mycobacterium tuberculosis complex in sputum or bronchial aspirate; compatible histopathological findings in lung tissue; and chest radiographic improvement after empirical TB treatment. The followup duration was defined as the time interval between enrolment and onset of TB treatment or, in the absence of $\mathrm{TB}$, the last chest radiograph within $1 \mathrm{yr}$. A grace period of 1 month after the study period was allowed to ascertain TB status within 1 yr. The TB notification registry was used to help track down all cases of active pulmonary TB.
Besides the primary end-point, the following data were collected: demographics, smoking and drinking history, past health, presenting symptoms, initial chest radiographic findings, initial sputum bacteriology results including AFB culture, clinical and chest radiographic progress within the initial 2 weeks up to 2 months, total number of sputum specimens and chest radiographs taken, antibiotics prescribed throughout the study period including fluoroquinolones other than allocated moxifloxacin, and the final diagnosis.

\section{Statistical analysis}

Assuming a Cohen's h value of 0.3 [17] for the difference in proportions of active pulmonary TB within $1 \mathrm{yr}$, a type I error of $5 \%$ by two-tailed analysis, and a statistical power of $80 \%$, the sample size per arm would be 175 patients. To allow for $20 \%$ loss to follow-up, we aimed at enrolling $\sim 210$ patients per arm. The primary end-point was analysed by modified intention to treat, which meant that all randomised participants would be analysed except for those with positive baseline AFB smear or culture. Besides comparing the proportion of the primary end-point in each treatment arm using statistical tests for categorical data, the Kaplan-Meier survival analysis was used to compare the time to the primary end-point using the log rank test. The Chi-squared test for trend, the log rank test for trend, and the likelihood ratio test statistic obtained from logistic risk models were used to examine whether there would be a dose-response relationship between the diagnosis of active pulmonary TB and moxifloxacin exposure. The Chi-squared test (with or without Yates' correction) or the Fisher's exact test was used to analyse categorical data. Two-sample t-tests or Mann-Whitney U-tests were used for examining continuous variables according to the data distribution pattern. One-way ANOVA or Kruskal-Wallis H tests were used for comparing continuous variables involving more than two groups. Statistical significance was taken as $p \leqslant 0.05$. All analyses were done with SPSS version 10 (SPSS, Inc., Chicago, IL, USA) and R version 2.9.0. This study was registered with www.hkclinicaltrials.com (number HKCTR-185).

\section{RESULTS}

From September 2004 to March 2007, a total of 550 patients were assessed for study eligibility. Figure 1 shows the flow of study from assessment of eligibility to analysis. After excluding 123 patients, 427 patients were enrolled and randomly assigned to receive either amoxicillin clavulanate $(n=212)$ or moxifloxacin $(n=215)$. Enrolled and excluded subjects were comparable by age $(\mathrm{p}=0.09$ by two-sample $\mathrm{t}$-test) and by sex $(\mathrm{p}=0.75$ by Chi-squared test).

Excluding three patients with positive baseline sputum culture, 424 participants were included in analysis by modified intention to treat. A total of $13(3.1 \%)$ patients developed active pulmonary TB: 10 out of 210 (4.8\% (95\% confidence interval (CI) $2.6-8.5 \%)$ ) were given amoxicillin clavulanate, and three out of $214(1.4 \%(95 \%$ CI $0.5-4.0 \%))$ were given moxifloxacin. The difference was significant both by proportion $(p=0.045)$ and time-to-event analysis $(p=0.04)$. The prevalence of cultureproven pulmonary TB in the current cohort was $1.9 \%$.

Tables 1 and 2 show the baseline characteristics and progress of participants. Patients assigned to receive moxifloxacin were largely comparable with those assigned to receive amoxicillin 


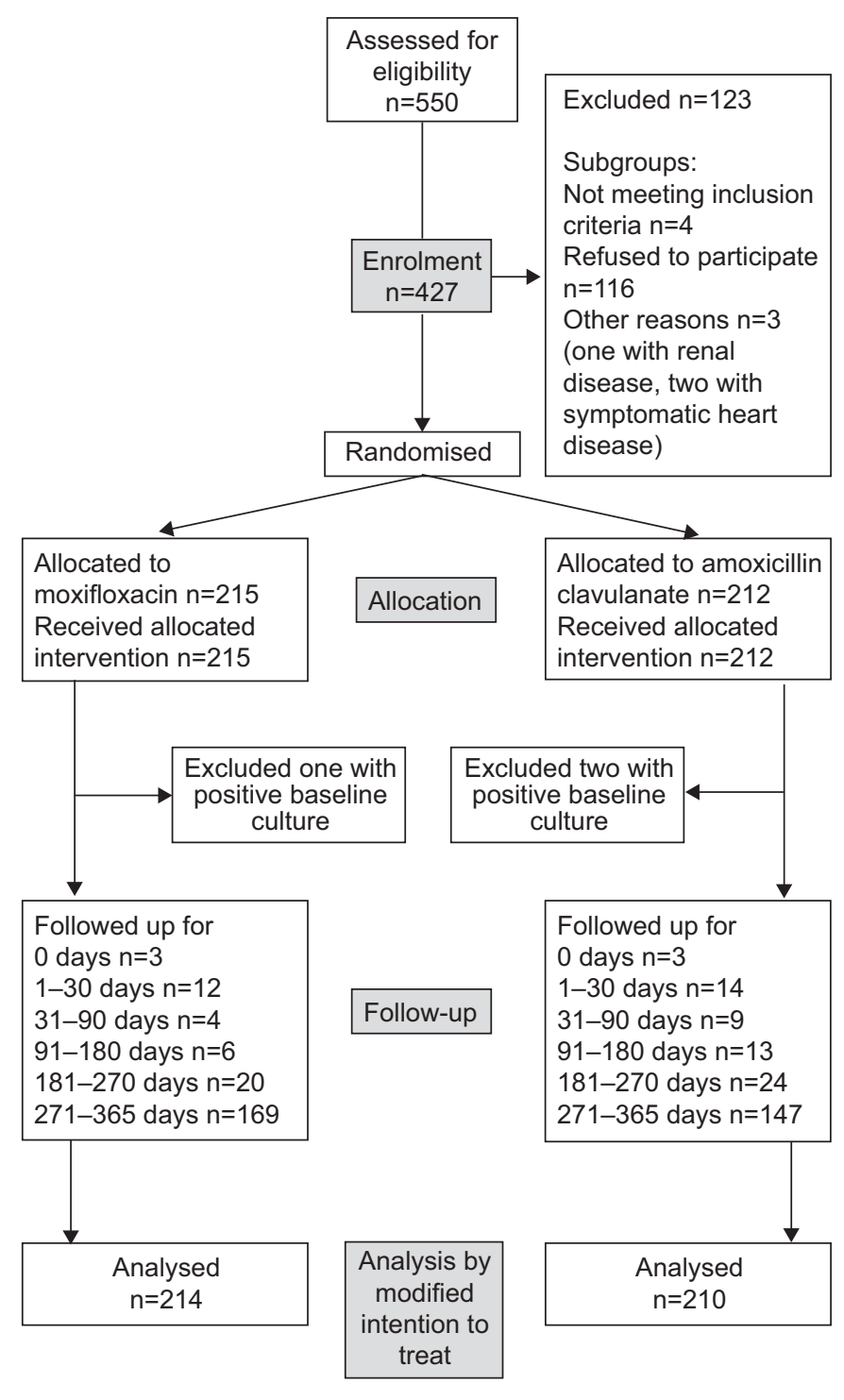

FIGURE 1. Flow chart from enrolment to analysis.

clavulanate with p-values $>0.10$ by statistical analysis except for previous TB treatment $(p=0.05)$ and chest pain $(p=0.04)$, neither of which were significantly associated with active pulmonary $\mathrm{TB}(\mathrm{p}=0.77$ and $\mathrm{p}=0.61$, respectively).

Table 3 compares TB cases by the treatment arm. Patients with active pulmonary $\mathrm{TB}$ among the moxifloxacin arm were all given moxifloxacin for 5 days. None of the three TB cases in the moxifloxacin arm was confirmed bacteriologically, whereas eight out of the $10 \mathrm{~TB}$ cases given amoxicillin clavulanate were culture-proven; the difference was significant $(p=0.04)$.

A total of $379(89.4 \%)$ and $360(84.9 \%)$ patients were followed up for at least 91 days and 181 days, respectively. Loss to follow-up as estimated by expected person-days over a period of 1 yr was $\sim 16 \%$ overall, $18 \%$ for participants in the amoxicillin clavulanate arm, and $14 \%$ for the moxifloxacin arm. The annual incidence of active pulmonary TB per 100,000 persons $(95 \%$ CI) was 3,769 $(2,111-6,262)$ overall,
6,072 (3,117-10,773) for participants given amoxicillin clavulanate, and 1,664 (461-4,440) for participants given moxifloxacin. Using TB notification rates of Hong Kong in 2005 as the reference, the expected sex- and age-adjusted incidence rates of active pulmonary $\mathrm{TB}$ in the current cohort would be $240(22-1,108)$.

Two patients took fluoroquinolones before the diagnosis of culture-proven pulmonary TB. Both were allocated to amoxicillin clavulanate rather than moxifloxacin. One received ciprofloxacin $250 \mathrm{mg}$ twice daily for 2 weeks and $500 \mathrm{mg}$ twice daily for 1 week, 14 weeks and 1 week, respectively, before the sputum specimen that isolated M. tuberculosis was collected. The other received ciprofloxacin 250-500 mg twice daily for 2 weeks, and $500 \mathrm{mg}$ twice daily for 1 week, $\sim 31$ weeks and 6 weeks, respectively, before the culturepositive sputum specimen was collected. Drug susceptibility testing showed bacillary susceptibility to ofloxacin in both.

Adverse events occurred in 11 patients allocated to moxifloxacin as follows: hospitalisation for pneumonia and acute exacerbation of chronic obstructive pulmonary disease $(n=2)$, left pleural effusion $(n=1)$, and acute respiratory distress syndrome that also occurred within 2 weeks after receiving standard TB treatment $(n=1)$; dizziness $(n=2)$; gastrointestinal upset $(n=3)$; transient twitching of extremities plus slurring of speech $(n=1)$; and a bitter taste $(n=1)$. Of these 11 patients, moxifloxacin was suspended in four: three for gastrointestinal upset and one for dizziness. Adverse events occurred in six patients allocated to amoxicillin clavulanate: hospitalisation within 1 month after enrolment for pneumonia $(n=1)$ and hypoglycaemia $(n=1)$; vague discomfort $(n=2)$; diarrhoea $(n=1)$; and transient chills in extremities $(n=1)$. Of these six patients, amoxicillin clavulanate was suspended in two patients for ill-defined symptoms (feeling hot and irritable) and generalised vague discomfort.

\section{Post hoc analysis of dose-response relationship}

The significant negative association between active pulmonary TB and exposure to moxifloxacin prompted a closer examination for any dose-response relationship among three subgroups of participants with different moxifloxacin exposure. The proportion with active pulmonary TB decreased significantly from $4.8 \%$ among participants given amoxicillin clavulanate $(n=210)$ to $2.4 \%$ and $0 \%$, respectively, among those given moxifloxacin for $5-7$ days $(n=127)$ and $10-15$ days within 4 weeks $(n=87 ; p=0.03)$. The log rank test for trend also showed a significant difference between the three subgroups $(p=0.02)$.

Online supplementary tables E1 and E2 compare baseline characteristics and progress of participants allocated to amoxicillin clavulanate $(n=210)$, moxifloxacin for 5-7 days $(n=127)$ and moxifloxacin for 10-15 days within 4 weeks $(n=87)$. The three groups were largely comparable, with p-values $>0.10$ by statistical analysis except for the following factors: previous TB treatment $(p=0.04)$, history of bronchiectasis $(p=0.07)$, chest pain $(p=0.09)$, fever $(p=0.01)$, symptomatic response within the initial 2 months $(p=0.01)$, and chest radiographic progress within the initial 2 months $(p=0.05)$. None of these factors were significantly associated with active pulmonary TB except for the last two $(p=0.001$ and $p<0.001$, respectively). 
TABLE 1 Baseline characteristics and treatment

\begin{tabular}{|c|c|c|}
\hline Variables & $\begin{array}{l}\text { Amoxicillin } \\
\text { clavulanate }\end{array}$ & Moxifloxacin \\
\hline Subjects $\mathbf{n}$ & 210 & 214 \\
\hline Male & $141(67.1)$ & $139(65.0)$ \\
\hline Age yrs & $64.6 \pm 13.7$ & $64.9 \pm 13.7$ \\
\hline Chinese & 209 (99.5) & $209(97.7)$ \\
\hline History of smoking & $97(46.2)$ & 99 (46.3) \\
\hline History of habitual drinking & $15(7.1)$ & $12(5.6)$ \\
\hline Previous TB treatment & 69 (32.9) & $90(42.1)$ \\
\hline History of bronchiectasis & $86(41.0)$ & $88(41.1)$ \\
\hline Diabetes mellitus & $13(6.2)$ & $15(7.0)$ \\
\hline Symptomatic on presentation & $198(94.3)$ & $194(90.7)$ \\
\hline \multicolumn{3}{|l|}{ Duration of symptoms } \\
\hline$<2$ weeks & $118(56.2)$ & $120(56.1)$ \\
\hline 2-4 weeks & $10(4.8)$ & $20(9.3)$ \\
\hline $1-2$ months & $38(18.1)$ & $35(16.4)$ \\
\hline$>2$ months & $31(14.8)$ & 27 (12.6) \\
\hline Indefinite & $13(6.2)$ & $12(5.6)$ \\
\hline Cough & $182(86.7)$ & $179(83.6)$ \\
\hline Sputum & $159(75.7)$ & $175(81.8)$ \\
\hline Haemoptysis & $48(22.9)$ & $41(19.2)$ \\
\hline Shortness of breath & $21(10.0)$ & $21(9.8)$ \\
\hline Chest pain & $21(10.0)$ & $10(4.7)$ \\
\hline Fever & $21(10.0)$ & $26(12.1)$ \\
\hline Weight loss & $9(4.3)$ & $14(6.5)$ \\
\hline \multicolumn{3}{|l|}{$\begin{array}{l}\text { Organisms isolated in sputum } \\
\text { bacterial culture }\end{array}$} \\
\hline Commensals & $142(67.6)$ & $135(63.1)$ \\
\hline Contaminants/colonisers & $8(3.8)$ & $18(8.4)$ \\
\hline Haemophilus influenzae & $23(11.0)$ & $21(9.8)$ \\
\hline $\begin{array}{l}\text { Streptococcus pneumoniae including } \\
\text { one case with Staphylococcus aureus }\end{array}$ & $7(3.3)$ & $5(2.3)$ \\
\hline Klebsiella pneumoniae & $3(1.4)$ & $6(2.8)$ \\
\hline Pseudomonas aeruginosa & $17(8.1)$ & $18(8.4)$ \\
\hline Others & $10(4.8)$ & $11(5.1)$ \\
\hline \multicolumn{3}{|l|}{ Change on initial $C \mathbf{R}^{\#}$} \\
\hline Lower zones only & $110(52.4)$ & $126(58.9)$ \\
\hline Upper or mid zones & $72(34.3)$ & $63(29.4)$ \\
\hline No change & $16(7.6)$ & $14(6.5)$ \\
\hline Uncertain & $12(5.7)$ & $11(5.1)$ \\
\hline \multicolumn{3}{|l|}{ Cavitations on initial CXR } \\
\hline No & $187(89.0)$ & $189(88.3)$ \\
\hline Yes & $7(3.3)$ & $11(5.1)$ \\
\hline No change & $16(7.6)$ & $14(6.5)$ \\
\hline \multicolumn{3}{|l|}{ Extent on initial CXR } \\
\hline$\leqslant$ equivalent of RUL & $189(90.0)$ & $195(91.1)$ \\
\hline > equivalent of RUL & $5(2.4)$ & $5(2.3)$ \\
\hline No change & $16(7.6)$ & $14(6.5)$ \\
\hline $\begin{array}{l}\text { Fluoroquinolones within } 4 \text { weeks } \\
\text { before enrolment }\end{array}$ & $1(0.5)$ & $1(0.5)$ \\
\hline \multicolumn{3}{|l|}{$\begin{array}{l}\text { Timing of first submission of sputum } \\
\text { for AFB bacteriology }\end{array}$} \\
\hline $0-1$ day & $133(63.3)$ & $147(68.7)$ \\
\hline$>1$ day & $76(36.2)$ & $67(31.3)$ \\
\hline Not submitted & $1(0.5)$ & $0(0)$ \\
\hline \multicolumn{3}{|l|}{ Duration of allocated antibiotics } \\
\hline 5 days & $123(58.6)$ & $118(55.1)$ \\
\hline
\end{tabular}

\begin{tabular}{lcc} 
TABLE 1 Continued & & \\
\hline Variables & $\begin{array}{c}\text { Amoxicillin } \\
\text { clavulanate }\end{array}$ & Moxifloxacin \\
\hline 7 days & $10(4.8)$ & $9(4.2)$ \\
10 days & $42(20.0)$ & $42(19.6)$ \\
Two 5-day courses with 2 weeks & $28(13.3)$ & $33(15.4)$ \\
Others" & $7(3.3)$ & $12(5.6)$ \\
\hline
\end{tabular}

Data are presented as $n(\%)$ or mean $\pm S D$, unless otherwise indicated. All comparisons show no significant difference (with $p>0.10$ ) except for previous tuberculosis (TB) treatment $(p=0.05)$ and chest pain $(p=0.04)$. Results that were unavailable or not applicable were excluded from statistical analysis. CXR: chest radiograph; RUL: right upper lobe; AFB: acid-fast bacilli. " ${ }^{\text {: }}$ with reference to previous films or those in the initial 2 months. Uncertainty of change was due to absence of previous films and no change in the initial 2 months. ": among patients allocated to receive amoxicillin clavulanate, five had two 5-day courses within 3-6 weeks, one had three 5-day courses within $\sim 2$ weeks, and one had four 5-day courses within 3 weeks. Among patients allocated to receive moxifloxacin, five had two 5-day courses within 3-4 weeks and seven had three 5-day courses within 2-4 weeks.

To further examine the dose-response relationship between moxifloxacin exposure and active pulmonary $\mathrm{TB}$, we tested the null hypothesis that the exposure effect of moxifloxacin was linear by comparing a logistic risk model that assumed a linear effect and a more general model in which the exposure effect was assumed to be non-linear. Ten covariates were included in the model: allocated antibiotics (amoxicillin clavulanate, moxifloxacin for 5-7 days, and moxifloxacin for 10-15 days), fluoroquinolones other than allocated moxifloxacin, sex, age, history of drinking, past TB treatment, history of bronchiectasis, presence or absence of symptoms on presentation, and cavitation and extent of disease on initial chest radiographs. Significant collinearity was excluded. The null hypothesis could not be rejected (likelihood ratio test statistic $=1.40$; $\mathrm{p}=0.24$ ). Findings reaffirmed a dose-response relationship between moxifloxacin exposure and diagnosis of active pulmonary TB. Table 4 shows a logistic risk model that assumes a linear effect for moxifloxacin exposure. The adjusted odds ratio $(95 \% \mathrm{CI})$ of active pulmonary $\mathrm{TB}$ after moxifloxacin exposure up to predominantly 10 days over 4 weeks was $0.3(0.1-0.9)$, or $0.9(0.3-3.0)$ after exposure for 1 day. Logistic risk models have been used instead of Cox models because coefficients failed to converge during Cox regression analysis.

\section{DISCUSSION}

The overall annual incidence rate of active pulmonary TB in the current cohort was $\sim 16$ times that of the sex- and age-adjusted TB notification rates of Hong Kong in 2005. The observed prevalence $(1.9 \%)$ of culture-proven active pulmonary TB in the current cohort was comparable with that observed in another local cohort hospitalised for CAP [18]. Patients given amoxicillin clavulanate had a significantly higher proportion of active pulmonary $\mathrm{TB}$ within $1 \mathrm{yr}$ than those who were given moxifloxacin $(p=0.045)$. Post hoc analysis showed a significant decrease in the proportion with active pulmonary TB from $4.8 \%$ 


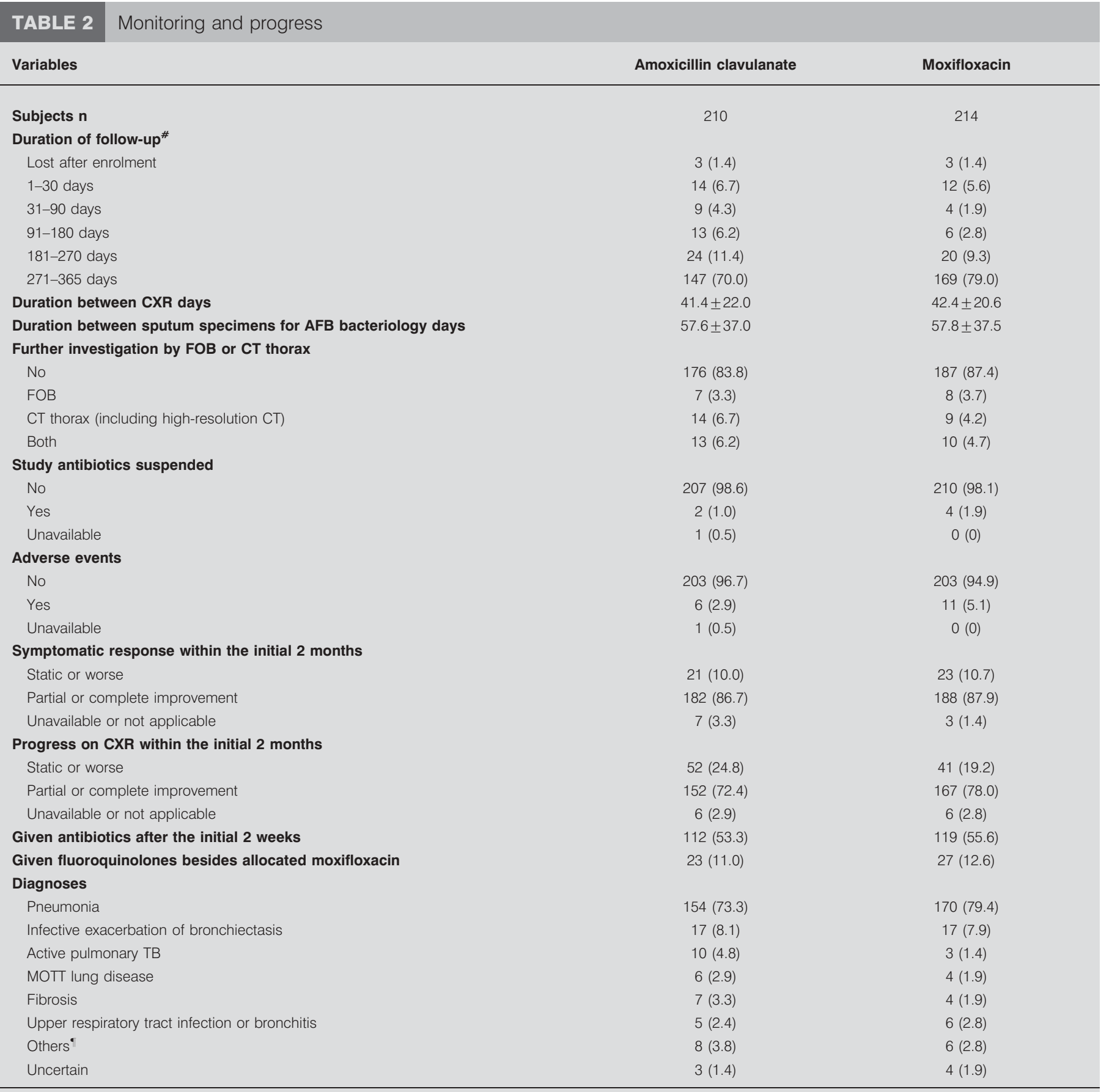

Data are presented as $\mathrm{n}(\%)$ or mean $\pm \mathrm{SD}$, unless otherwise indicated. All comparisons show no significant difference (with $\mathrm{p}>0.10$ ). Results that were unavailable or not applicable were excluded from statistical analysis. CXR: chest radiograph; AFB: acid-fast bacilli; FOB: fibreoptic bronchoscopy; CT: computerised tomography; TB: tuberculosis; MOTT: mycobacteria other than TB. ${ }^{*}$ : defined as the interval between enrolment and TB treatment within $1 \mathrm{yr}$ or, in the absence of TB treatment, the last CXR within $1 \mathrm{yr}$. A grace period of 1 month after the study period was allowed to ascertain TB status within $1 \mathrm{yr}$. ": others in the moxifloxacin arm included two cases of lung cancer, and one case each of sputum atypia, probably malignancy, Churg-Strauss syndrome, and idiopathic pulmonary fibrosis. Others in the amoxicillin clavulanate arm referred to eight cases of lung cancer.

among participants given amoxicillin clavulanate to $2.4 \%$ and $0 \%$ among those given moxifloxacin for predominantly 5 days and predominantly 10 days within 4 weeks, respectively $(p=0.03$ by Chi-squared test for trend). The linear effect was reaffirmed in regression models. A logistic risk model with a linear effect for moxifloxacin exposure showed that the adjusted odds ratio ( $95 \% \mathrm{CI}$ ) of active pulmonary TB after moxifloxacin exposure up to predominantly 10 days over 4 weeks was $0.3(0.1-0.9)$, or $0.9(0.3-3.0)$ after exposure for 1 day. 
TABLE 3 Comparing patients with active pulmonary tuberculosis (TB) in the two treatment arms

\begin{tabular}{|c|c|c|c|}
\hline & Amoxicillin clavulanate & Moxifloxacin & p-value \\
\hline Subjects $\mathbf{n}$ & 10 & 3 & \\
\hline Male & $9(90.0)$ & $2(66.7)$ & $>0.2$ \\
\hline Age yrs & $67.7(33.5-84.4)$ & $70.2(24.6-71.6)$ & $>0.2$ \\
\hline Symptomatic on presentation & $7(70.0)$ & $2(66.7)$ & $>0.2$ \\
\hline Cavities & $2(20)$ & $0(0)$ & $>0.2$ \\
\hline Culture-proven TB & $8(80)$ & $0(0)$ & 0.04 \\
\hline First sputum submitted within 1 day & $2(20.0)$ & $2(66.7)$ & $>0.2$ \\
\hline Time of collection of first positive culture days & $21(5-282)$ & NA & NA \\
\hline Onset of TB treatment days & $23.5(6-360)$ & $12(7-137)$ & $>0.2$ \\
\hline Potential masking of TB through improvement in symptoms within the initial 2 months & $4(44.4)$ & $2(66.7)$ & $>0.2$ \\
\hline
\end{tabular}

With less error due to selection bias and confounding in comparison with retrospective studies, the current randomised controlled trial has put the risk of using fluoroquinolones for treating community-acquired respiratory infection into a better perspective. Dooley et al. [14] showed that initial empirical therapy with fluoroquinolones was associated with a delay in TB treatment (21 days versus 5 days). In a retrospective analysis involving $548 \mathrm{~TB}$ patients, WANG et al. [19] demonstrated that exposure to fluoroquinolones delayed TB treatment and was associated with poor prognosis. Our findings show that newer fluoroquinolones, such as

\section{TABLE 4 A logistic risk model that assumes a linear effect} for moxifloxacin exposure

\begin{tabular}{|c|c|c|}
\hline Predictor variables & OR $(95 \% \mathrm{Cl})$ & p-value \\
\hline Moxifloxacin exposure & $0.3(0.1-0.9)$ & 0.03 \\
\hline $\begin{array}{l}\text { Fluoroquinolones other } \\
\text { than allocated moxifloxacin }\end{array}$ & $2.6(0.4-17.3)$ & 0.33 \\
\hline Male & $2.1(0.4-11.7)$ & 0.41 \\
\hline Age yrs & $0.97(0.93-1.01)$ & 0.16 \\
\hline History of drinking & $2.8(0.5-15.8)$ & 0.24 \\
\hline Past TB treatment & $0.6(0.1-2.4)$ & 0.43 \\
\hline History of bronchiectasis & $0.4(0.1-2.1)$ & 0.30 \\
\hline Symptomatic on presentation & $0.1(0.0-0.5)$ & 0.01 \\
\hline $\begin{array}{l}\text { Cavitation on initial } \\
\text { chest radiograph }\end{array}$ & $4.0(0.5-34.8)$ & 0.22 \\
\hline $\begin{array}{l}\text { Extent of disease greater than } \\
\text { equivalent of right upper } \\
\text { lobe on chest radiograph }\end{array}$ & $29.5(4.3-203.1)$ & $<0.001$ \\
\hline
\end{tabular}

History of smoking has been excluded owing to significant correlation with males. TB: tuberculosis. moxifloxacin, appear to mask TB in proportion to the duration of exposure in TB-endemic areas.

Allowing use of nonstudy antibiotics and flexibility in the dosing duration of study antibiotics and frequency of chest radiographic examination and sputum AFB bacteriology probably rendered the current trial more like a real-life situation. Despite the lack of a double-blind placebo-controlled design, randomisation was probably adequate, as shown by tables 1 and 2. It might be argued that in the absence of blinding, the attending physician might be prone to start empirical TB treatment in the moxifloxacin arm. If this were the case, more patients in the moxifloxacin arm would have received TB treatment. There were in fact more $\mathrm{TB}$ cases among patients allocated to receive amoxicillin clavulanate.

Although a dose-response relationship between moxifloxacin and masking of TB could be reaffirmed in the current study through regression models, the linear effect might have been better shown without regression analysis had the study design included two moxifloxacin arms with different treatment durations allocated by randomisation. Symptomatic response and chest radiographic progress within the initial 2 months were deliberately excluded from regression analysis despite their significant association with both moxifloxacin exposure and active pulmonary $\mathrm{TB}$; inclusion would have been inappropriate as moxifloxacin probably masks TB through improvement in symptoms or chest radiography [20].

The small number of TB cases made it difficult for the current trial to address the association between newer fluoroquinolones and culture-negative pulmonary TB. Although a retrospective study showed no significant association between fluoroquinolone exposure and an increased risk of culture-negative TB [21], further studies are warranted to examine whether prolonged or multiple courses of newer fluoroquinolones may reduce sputum culture yield of active pulmonary TB. 
Another concern about treating community-acquired chest infection with fluoroquinolones is the emergence of fluoroquinoloneresistant TB. The small number of study participants with exposure to fluoroquinolones before a culture-positive sputum specimen was collected made it difficult for the current trial to address this issue. However, a number of studies have reported a low risk of fluoroquinolone-resistant $\mathrm{TB}$ among patients exposed to fluoroquinolones prior to the diagnosis of TB [22-24]. HUANG et al. [25] found that resistance to fluoroquinolones was probably due to use of fluoroquinolones among patients with multidrugresistant TB rather than the general community. It has also been suggested that newer fluoroquinolones with better pharmacokinetic and pharmacodynamic properties help reduce the risk of developing drug resistance in $M$. tuberculosis [26]. Some preliminary evidence in that direction regarding high-dose moxifloxacin and levofloxacin were indeed obtained [27, 28]. However, the safety and tolerance of these higher dosages require further exploration [27]. Current data also favour shortening the duration of antibiotic treatment at an optimal dosage to reduce the risk of acquired resistance [24, 29]. In corroboration with recent findings of an association between fluoroquinoloneresistant TB and prolonged or multiple courses of fluoroquinolones $[22,30]$, the current study would caution against fluoroquinolone use in excess of 5 days or multiple courses in TB-endemic areas to reduce the risk of masking active TB alongside that of developing fluoroquinolone-resistant TB.

The impact of HIV infection on the findings of our clinical trial could not be examined because the prevalence of HIV in Hong Kong was $<1 \%$ [15]. As the clinical and radiographic presentation of pulmonary TB in HIV-infected subjects become atypical with immunosuppression, whether findings of the current trial may also apply to areas that are endemic for both TB and HIV is uncertain.

In conclusion, newer fluoroquinolones, such as moxifloxacin, appear to mask active pulmonary $\mathrm{TB}$ in proportion to the duration of exposure in TB-endemic areas.

\section{CLINICAL TRIALS}

This study was registered with www.hkclinicaltrials.com (number HKCTR-185).

\section{STATEMENT OF INTEREST}

None declared.

\section{ACKNOWLEDGEMENTS}

The authors would like to extend their gratitude to all nurses of the Tuberculosis and Chest Service of the Dept of Health, Hong Kong Special Administrative Region, for their invaluable help. Special thanks go to L. Ku for coordinating the study, and I. Mak and B. Wong for data entry (all Tuberculosis and Chest Service of the Dept of Health, Hong Kong Special Administrative Region).

\section{REFERENCES}

1 Lode H, Garau J. Improving care for patients with respiratory tract infections. J Chemother 2002; 14: Suppl. 2, 22-28.

2 Tillotson GS. Role of gemifloxacin in community-acquired pneumonia. Expert Rev Anti Infect Ther 2008; 6: 405-418.

3 Miravitlles M, Anzueto A. Moxifloxacin: a respiratory fluoroquinolone. Expert Opin Pharmacother 2008; 9: 1755-1772.
4 Vardakas KZ, Siempos II, Grammatikos A, et al. Respiratory fluoroquinolones for the treatment of community-acquired pneumonia: a meta-analysis of randomized controlled trials. CMAJ 2008; 179: 1269-1277.

5 Petitpretz P, Arvis P, Marel M, et al. Oral moxifloxacin vs. highdosage amoxicillin in the treatment of mild-to-moderate, community-acquired, suspected pneumococcal pneumonia in adults. Chest 2001; 119: 185-195.

6 Torres A, Muir JF, Corris P, et al. Effectiveness of oral moxifloxacin in standard first-line therapy in community-acquired pneumonia. Eur Respir J 2003; 21: 135-143.

7 Mandell LA, Wunderink RG, Anzueto A, et al. Infectious Diseases Society of America/American Thoracic Society consensus guidelines on the management of community-acquired pneumonia in adults. Clin Infect Dis 2007; 44: Suppl. 2, S27-S72.

8 Yew WW, Chan CK, Chau CH, et al. Outcomes of patients with multidrug-resistant pulmonary tuberculosis treated with ofloxacin/ levofloxacin-containing regimens. Chest 2000; 117: 744-751.

9 Yew WW, Chan CK, Leung CC, et al. Comparative roles of levofloxacin and ofloxacin in the treatment of multidrug-resistant tuberculosis: preliminary results of a retrospective study from Hong Kong. Chest 2003; 124: 1476-1481.

10 Chan ED, Laurel V, Strand MJ, et al. Treatment and outcome analysis of 205 patients with multidrug-resistant tuberculosis. Am J Respir Crit Care Med 2004; 169: 1103-1109.

11 Leimane V, Riekstina V, Holtz TH, et al. Clinical outcome of individualised treatment of multidrug-resistant tuberculosis in Latvia: a retrospective cohort study. Lancet 2005; 365: 318-326.

12 Rustomjee R, Lienhardt C, Kanyok T, et al. A phase II study of the sterilising activities of ofloxacin, gatifloxacin and moxifloxacin in pulmonary tuberculosis. Int J Tuberc Lung Dis 2008; 12: 128-138.

13 Conde MB, Efron A, Loredo $\mathrm{C}$, et al. Moxifloxacin versus ethambutol in the initial treatment of tuberculosis: a double-blind, randomised, controlled phase II trial. Lancet 2009; 373: 1183-1189.

14 Dooley KE, Golub J, Goes FS, et al. Empiric treatment of community-acquired pneumonia with fluoroquinolones, and delays in the treatment of tuberculosis. Clin Infect Dis 2002; 34: $1607-1612$.

15 Tuberculosis and Chest Service of the Dept of Health. Annual Report 2007. The Government of Hong Kong SAR.

16 Mandell LA, File TMJ. Short-course treatment of communityacquired pneumonia. Clin Infect Dis 2003; 37: 761-763.

17 Cohen J. Statistical Power Analysis for the Behavioral Sciences. Rev. Edn. New York, Academic Press, 1977.

18 Ko FWS, Lam RKY, Li TST, et al. Sputum bacteriology in patients hospitalized with acute exacerbations of chronic obstructive pulmonary disease and concomitant pneumonia in Hong Kong. Intern Med J 2005; 35: 661-667.

19 Wang J, Hsueh P, Jan I, et al. Empirical treatment with a fluoroquinolone delays the treatment for tuberculosis and is associated with a poor prognosis in endemic areas. Thorax 2006; 61: 903-908.

20 Rothman KJ. Epidemiology: An Introduction. New York, Oxford University Press, Inc., 2002.

21 Gaba PD, Haley C, Griffin MR, et al. Increasing outpatient fluoroquinolone exposure before tuberculosis diagnosis and impact on culture-negative disease. Arch Intern Med 2007; 167: 2317-2322.

22 Long R, Chong H, Hoeppner V, et al. Empirical treatment of community-acquired pneumonia and the development of fluoroquinolone-resistant tuberculosis. Clin Infect Dis 2009; 48: 1354-1360.

23 Ginsburg AS, Grosset JH, Bishai WR. Fluoroquinolones, tuberculosis, and resistance. Lancet Infect Dis 2003; 3: 432-442.

24 Ginsburg AS, Hooper N, Parrish N, et al. Fluoroquinolone resistance in patients with newly diagnosed tuberculosis. Clin Infect Dis 2003; 37: 1448-1452. 
25 Huang T, Kunin CM, Shin-Jung Lee S, et al. Trends in fluoroquinolone resistance of Mycobacterium tuberculosis complex in a Taiwanese medical centre: 1995-2003. J Antimicrob Chemother 2005; 56: 1058-1062.

26 Drlica K, Zhao X. Mutant selection window hypothesis updated. Clin Infect Dis 2007; 44: 681-688.

27 Gumbo T, Louie A, Deziel MR, et al. Selection of a moxifloxacin dose that suppresses drug resistance in Mycobacterium tuberculosis, by use of an in vitro pharmacodynamic infection model and mathematical modeling. J Infect Dis 2004; 190: 1642-1651.
28 Johnson JL, Hadad DJ, Boom WH, et al. Early and extended early bactericidal activity of levofloxacin, gatifloxacin and moxifloxacin in pulmonary tuberculosis. Int J Tuberc Lung Dis 2006; 10: 605-612.

29 Low DE. Fluoroquinolones for treatment of community-acquired pneumonia and tuberculosis: putting the risk of resistance into perspective. Clin Infect Dis 2009; 48: 1361-1363.

30 Devasia RA, Blackman A, Gebretsadik T, et al. Fluoroquinolone resistance in $M$. tuberculosis: the effect of duration and timing of fluoroquinolone exposure. Am J Respir Crit Care Med 2009; 180: 365-370. 